\title{
Novel Dynamic Technique Reducing the Offset Voltage in a Hall Effect Sensor
}

\author{
Vlassis Petoussis, Panos Dimitropoulos, Gerorge Stamoulis, Elias Houstis \\ University of Thessaly, Department of Computer \& Communications Engineering \\ 37th Glavani \& 28th October Str ,Volos, Greece
}

\begin{abstract}
Silicon Hall plates show an offset of a few millitesla. A large portion of this offset is caused by mechanical stress in the device. The offset can be reduced with a factor $10^{3}$ to $10^{5}$ when the spinning - current principle is applied. In this paper we present a model and numerical analysis of a new Hall effect sensor which using a novel offset reduction method.

We call it "Wheel Hall Senor". The device exploits the signals attributed to Hall voltage, Hall current and geometric MR effect. As a result the device is equivalent to an "ideal" voltage or current Hall sensor with geometrical factor of one $\left(G_{H}=1\right)$. Moreover it provides for high-speed spinning, given that the voltage distribution changes moderately between different phases. This is equivalent to minimum charge injection that 0 in turn - allows spinning frequency increase. The device senses all 3 filed dimensions, namely the flux-density of $B_{Z}$ is proportional to the DC component of the output signal, whereas the $B_{X}$ and $B_{Y}$ components are proportional to the first harmonic of the output signal. Finally the device can be made in a way to reuse the current, if integrated in a BiCMOS technology providing for matched JFETs.

Furthermore we calculate the function which governs the changes in the electric field inside the new Hall effect sensor in presence of magnetic field. This function help us to control in MatLab environment the equipotential lines and to monitor the changes when biasing conditions are change. The combination of his pioneering form and the elaborate sequence of using the dynamic spinning current technique, leads to satisfactory results of produced Hall voltage with small noise in a presence of external magnetic field.
\end{abstract}

\section{Introduction}

Hall plates are used in a wide variety of applications to measure static and dynamic magnetic fields. A major drawback of these sensors is their high offset voltage, i.e., an output signal in the absence of a magnetic induction. According to [1] the effects which contribute to the offset voltage are piezoresistive effects, geometrical errors, temperature gradients, nonhomogeneities, etc. However, the offset voltage with a spatially periodic nature differs from the Hall voltage, which is constant. One way to reduce the offset caused by orthogonal asymmetries is to use coupled symmetric Hall plates [2]. However, the offset can never be completely cancelled because there will always be small differences between the Hall devices. To overcome the mismatch, the bias current of a single four contact Hall device is spun by contact commutating and the resulting output voltages are averaged over time. The orientation dependence of the offset sources in silicon suggests that orthogonal switching is not sufficient. To cancel components of higher asymmetries a multicontact Hall plate with more than four terminals is necessary. The method presented in this paper is based on Hall device with sixteen outside and sixteen inside contacts. The spinning current vector is generated by two harmonic biasing currents. As a result, the output signal becomes spatially continuously accessible, hence, asymmetries of higher order can be cancelled out. Aim of present work is the presentation of new Hall sensor with pioneering form that reminds cogwheel with two lines teeth, one internally and one externally (for this reason we named Wheel Hall Sensor) but also with different way in the cancellation of offset voltage and finally $1 / \mathrm{f}$ noise rejection. Initially the designing constituted fundamental objective that as aim had the respect and compatibility in the existing techniques of reduction $1 / \mathrm{f}$ noise and also offset voltage. For this aim was selected the circular structure.

\section{Offset voltage}

In a standard IC process Hall plates have the advantage of cheap and small. Therefore they are quite often used in applications where the permanent magnet is combined with the sensor. Because Hall plates show a large offset the magnet used in these applications have to be a strong magnet. The offset of the Hall plate is the voltage that is measured when no magnetic field is applied. Eq. 1 represents the theoretical situation, when an offset is added this equation becomes: 


$$
V_{H}=I S B+V_{o f f s e t}
$$

Where $\mathrm{I}$ is the biasing current and $\mathrm{S}$ is the current - related sensitivity. To reduce the offset, which is time variant, spinning current Hall plates was developed [3]. These are symmetrical Hall plates with more than 4 contacts. In this multi contact Hall plates the bias current is switched in for example, eight directions through the Hall plate and the corresponding voltages are measured, see Fig 1.[4].

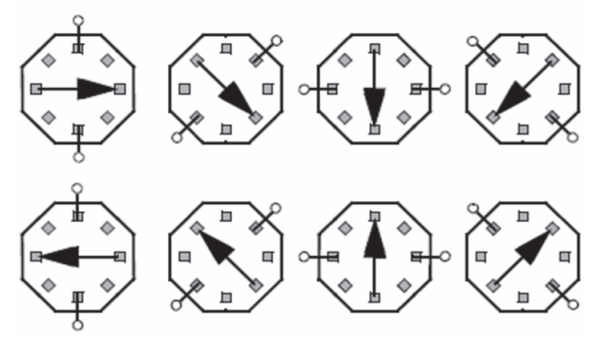

Figure. 1 An eight contact spinning-current Hall plate with the possible bias current directions

The measured voltages represent one period of a (radial spatial) periodic signal. The spinning current method uses this periodicity, it separates transduction effects according to their spatial radial periodicity. The Hall effect itself does not depend on bias current direction and is therefore present in the DC component. So, a spinning-current Hall plate solves one of the most important drawbacks in integrated silicon Hall plates, their offset.

\section{The split - current model}

In this section, we will represent the magnetic field resolution achievable with Hall split-current, sensor and the offset voltage for a Hall plate. The presented results have been checked modeling the Hall devices with an equivalent electrical circuit, consisting of a network of resistances and voltage controlled current sources, as proposed in $[5,6]$.

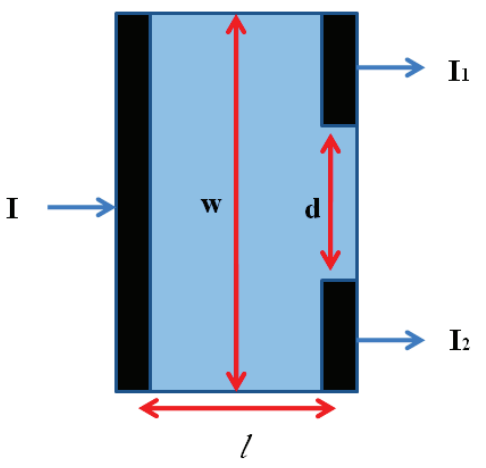

Figure.2 The split-current Hall effect device.

Fig. 2 shows the structure of a split-current device, placed in a static magnetic field $B=B \hat{z}$, and biased with a current $I$. The current difference between the two split contacts is [3]

$$
\Delta I=F \mu I B
$$

where $I$ and $w$ are the length and width of the device, $d$ is the distance between the split contacts, and $F$ is a magneto-geometrical factor.

\section{A set of equations monitor the changes of the electric field inside the sensor}

In this section we calculate a set of functions which governs the changes in the electric field inside the new Hall effect sensor in presence of magnetic field $\mathbf{B}$. This function help us to control in MatLab 
environment the equipotential lines and to monitor the changes on them when biasing conditions are change. In our split-current model we use Dirichlet and Neumann boundary conditions as we can see in Fig.3. Use the following empirical expressions [7]

$$
\begin{aligned}
& \mu_{n}=\left(65.0+\frac{1265}{1+\left[N_{T} /\left(8.5 \times 10^{16} \mathrm{~cm}^{-3}\right)\right]^{0.72}}\right) \frac{\mathrm{cm}^{2}}{V_{S}} \\
& \mu_{p}=\left(46.7+\frac{447.3}{1+\left[N_{T} /\left(6.3 \times 10^{16} \mathrm{~cm}^{-3}\right)\right]^{0.72}}\right) \frac{\mathrm{cm}^{2}}{V_{S}}
\end{aligned}
$$

where $\mathrm{N}_{\mathrm{T}}$ denotes the total ionized impurity concentration. For the Hall mobility we use the approximation [8] $\mu^{*}=r \mu$, with $r=\left\langle T^{2}\right\rangle \mid\langle T\rangle^{2}$ [9]. We consider semiconductor slabs of rectangular geometry with a magnetic field perpendicular to the device surface: $\mathbf{B}=(0,0, B$,$) . The distributions of carriers and the$ potential in the device depend only on $\mathrm{x}$ and $\mathrm{y}$ and we solve a two-dimensional problem.

$$
\begin{array}{r}
\frac{\partial \Phi}{\partial x}=-c(B) \frac{\mu_{p} B}{1+\left(\mu_{p} B\right)^{2}} \quad \begin{array}{c}
\frac{\partial \Phi}{\partial x}=c(B) \frac{\mu_{n} B}{1+\left(\mu_{n} B\right)^{2}} \\
c(B)=-\frac{J}{\sigma_{p B}} \hat{y}
\end{array}
\end{array}
$$

And we get two equations (Eq.5) for a $\mathrm{p}$ and $\mathrm{n}$ type respectively. These equations give us the dependence of the electric field inside the sensor in presence of magnetic field.

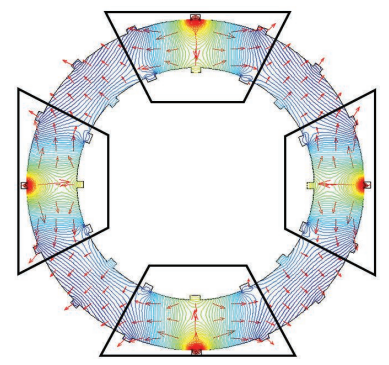

(a)

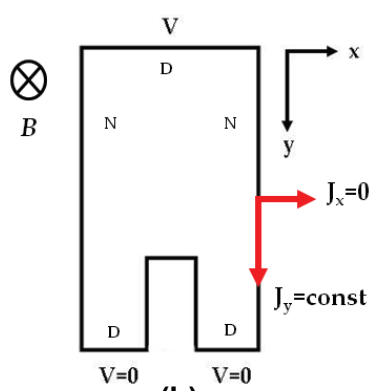

(b)

Figure. 3 The boundary conditions for a split-current element Hall effect device. a) Graphic representation of a simple model in even phase, with $\mathrm{D}$ and $\mathrm{N}$ presented the boundary conditions. Dirichlet boundary conditions in contacts with positive or zero potential and Neumann boundary conditions elsewhere. b) The area of the received Hall voltage tacked in four places of the sensor. We can see that the margins of the field in our model is well separated each other (black trapezoid areas).

\section{The Wheel Hall Sensor (WHS)}

A novel Hall sensor device introduced which uses elaborate spinning current technique. The novel Hall device that we call "Wheel Hall Senor" is presented in Figures 4a and 4b. The current enters the device, as presented in the aforementioned Figures, in two phases namely the even phase (PHASE-P) and the odd phase (PHASE-N). The device exploits the signals attributed to Hall voltage, Hall current and geometric MR effect. As a result the device is equivalent to an "ideal" voltage or current Hall sensor with geometrical factor of one $\left(G_{H}=1\right)$. Moreover it provides for high-speed spinning, given that the voltage distribution changes moderately between different phases. This is equivalent to minimum charge injection that 0 in turn - allows spinning frequency increase. The device senses all 3 filed dimensions, namely the flux-density of $B_{Z}$ is proportional to the DC component of the output signal, whereas the $B_{X}$ and $B_{Y}$ components are proportional to the first harmonic of the output signal. Finally the device can be made in a way to reuse the current, if integrated in a BiCMOS technology providing for matched JFETs. 


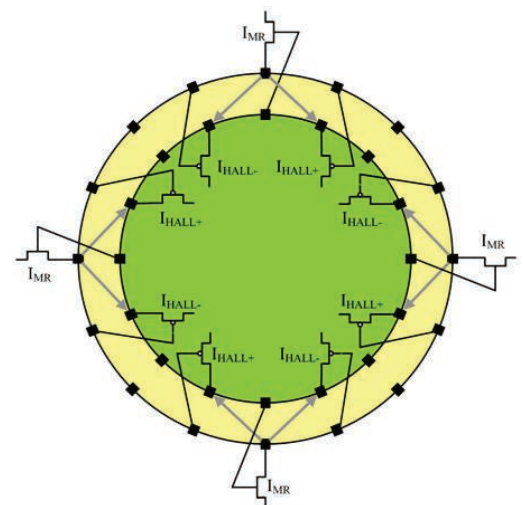

(a)

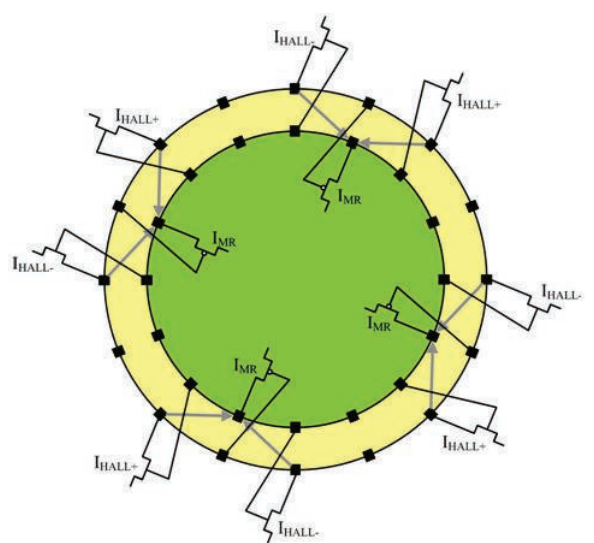

(b)

Figure. 4 The novel Hall device that we call "Wheel Hall Senor" or WHS. (a) The even phase (PHASE-P) ; (b) the odd phase (PHASE-N).

\section{The offset reduction method}

To reduce the offset, the new Hall effect sensor uses spinning-current technique in the symmetrical Hall plate (Fig.4). In the offset reduction sequence the direction of the bias current is splited right and left and the corresponding output voltage is measured on the contacts in $\pm 45^{\circ}$ to the current direction in each phase. The total number of measurements at each phase is in four places like a cross and all phases give as the total Hall voltage. When sixteen outside and sixteen inside contacts are used, the bias current is switched $\pm 45^{\circ}$ for each measurement, the voltage contacts are switched outside to inside respectively (Fig. 5). For each phase of the bias current, four output $\left(\mathrm{V}_{\mathrm{H}_{+}}\right.$and $\left.\mathrm{V}_{\mathrm{H}_{-}}\right)$and input $\left(\mathrm{V}_{\mathrm{H}_{-}}\right.$and $\left.\mathrm{V}_{\mathrm{H}_{+}}\right)$voltages are measured in a rotating clock wise. Finally for each turn in PHASE-P and PHASE-N, totally sixteen Hall voltages are measured. Each harmonic biasing current in each phase produces an offset voltage witch totally in turns gives us the offset cancellation. So offset caused from current $I_{A D}$ in phase $P$ cancel the offset caused from current $I_{C D}$ in phase $N$ and $I_{A D}$ in phase $N$ cancel the offset caused from current $I_{A F}$ in phase P. Finally the one offset in one phase cancel the other in next phase [10].

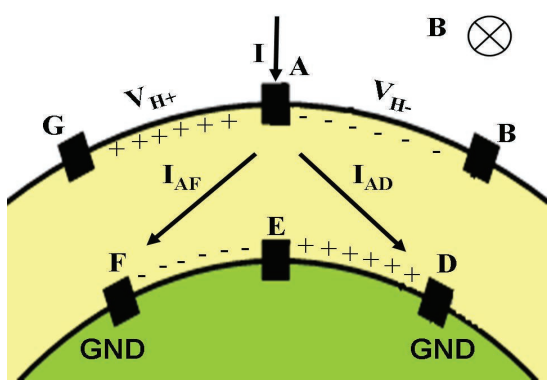

(a)

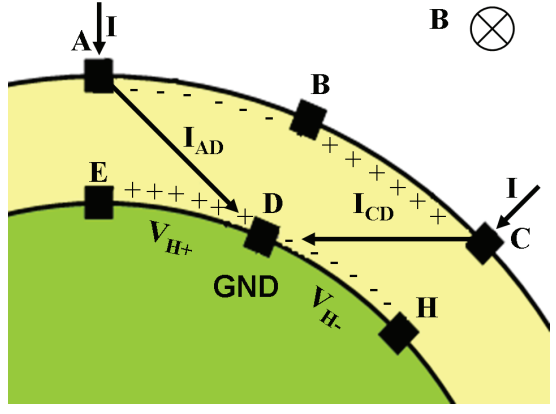

(b)

Figure. 5 The even phases (a) and the odd phases (b). With I we denote the current bias in $45^{\circ}$ direction in each measurement and $\mathrm{V}_{\mathrm{H}^{+}}$and $\mathrm{V}_{\mathrm{H}}$ - denoted the two Hall Voltage references in each phase (for $\mathrm{n}$-type material).

\section{The real structure of the sensor}

The main shape of the sensor is a circle (Fig.4). This shape to be in real material contains problems especially on the offset caused by stress. Furthermore the designed shape must satisfy a way to be easy functionally for a spinning-current technique. The final decision was to develop the sensor in a row with a two lines of small Hall Effect devices with up and down parts in a reversed trapezoid shape resemble the real cycled structure of the sensor. We need totally 144 P-N junction diffusions pieces (Fig.6) divided in 32 triple trapezoid groups, each group has a contact for measuring or biasing and finally we use 32 contacts. 

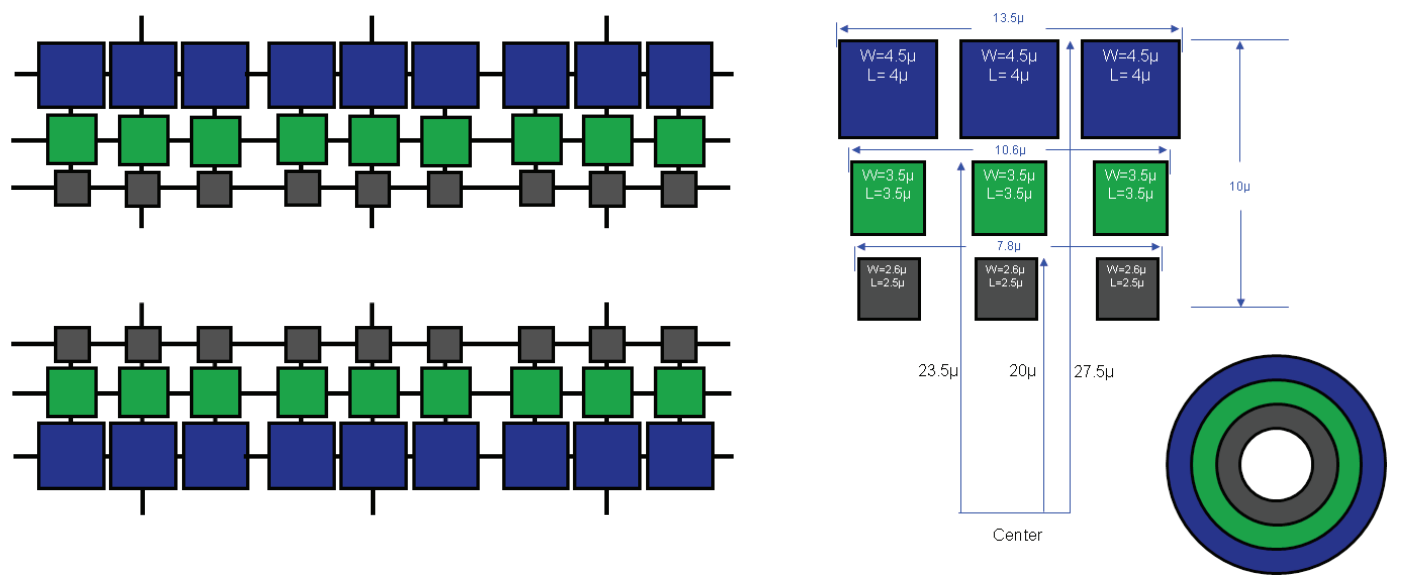

Figure. 6 The real structure of the "Wheel Hall Sensor". We see only a part of the 144 P-N junction diffusions divided in triple groups, each group has a contact for measuring and biasing. A triple group of P-N junction diffusion with proposed dimensions.

\section{Field Simulations}

In this part we present simulations of the device structure with a MatLab simulator. The main goal was to see the behavior of the electric field inside the device when this exposed in external magnetic field Fig( $7 a$, $7 \mathrm{~b})$. In a way to control the equipotential lines in it, we solve the Laplace equation using the boundary conditions that we saw in the split current model previously. The results show us that the shape and the place of the contacts is in the right position and are well combined in even and odd phase during the spinning current technique.

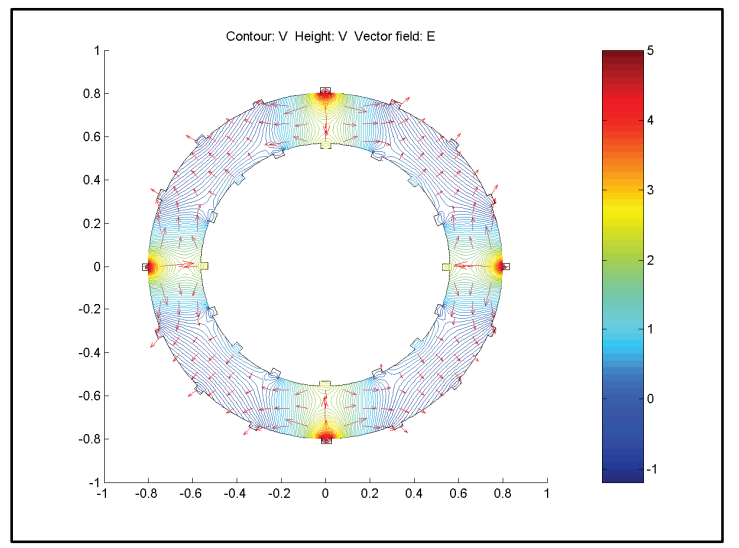

(a)

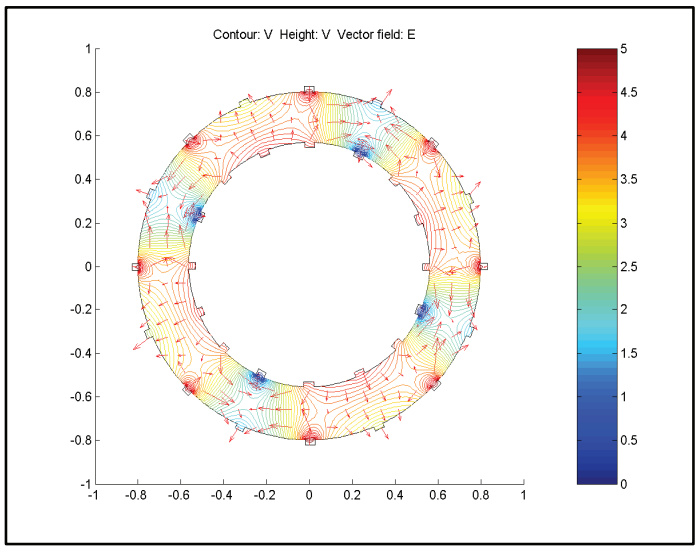

(b)

Figure. 7 The novel Hall device that we call "Wheel Hall Senor" or WHS. (a) The electric field and the equpotential lines in the even phase (PHASE-P) for $B \neq 0$; (b) The electric field and the equpotential lines in the odd phase (PHASE-N) for $\mathrm{B} \neq 0$.

\section{Acknowledgments}

I would like to thank Pr. Dr. George Stamoulis, Dr. Panos Dimitropoulos for the useful advices and Pr.Dr. Manolis Vavalis for the helpful discussions. Furthermore I would like to thank the Electrical Engineering \& Computer Engineering Department of the University of Thessaly for the technical support and fulfillment of this project. 


\section{References}

[1] A. A. Bellekom and P. J. A. Munter, "Offset reduction in spinning current Hall plates," Sensor and Materials 5,253- 263, 1994.

[2] J. Maupin and M. Geske, "The Hall effect in silicon circuits," The Hall effect and its applications, ed. C. Chien and C. Westgate, New York, 1980.

[3] R.S. Popovic, Hall Effect Devices, Adam Hilger, Bristol, 1991.

[4] Sandra Bellekom, Lina Sarro. International Conference on Solid-State Sensors and Actuators, Chicago, June 16-19, 1997 p.233-p236.

[5] C. Maier, M. Emmenegger, S. Taschini, H. Baltes, J.G. Korvink, Equivalent circuit model of resistive IC sensors with the box integration method, IEEE Trans. Comput.-Aided Des. Integr. Circuits Syst. 18 (1999) 1000-1013.

[6] R.S. Popovic, Numerical analysis of MOS magnetic field sensors, Solid State Electron. 28 (1985) 711-716.

[7] A. W. Vinal and N. A. Masnari, "Magnetic transistor behav:cslr explained by modulation of emitter injection, not carrier deflection," IEEE Electron Device Lett., vol. EDL-3, pp. 203-2C 5,1982.

[8] R. A. Smith, Semiconductors. New York: Cambridge, 1978.

[9] A. W. Vinal and N. A. Masnari, 'Response to "Comment on 'Magnetic transistor behavior explained by modulation of emitter injection, not carrier deflection,' IEEE Electron Device Lett . vol.EDL ,pp. 396-397, 1982

[10] Vlassis N. Petoussis , P.Dimitropoulos, George Stamoulis, A Novel Hall Effect Sensor Using Elaborate Offset Cancellation Method, Sensors \& Transducers Journal, Vol. 100, Issue 1, January 2009, pp. 85-91. 